These efforts have reduced unnecessary patient room entry, minimized HCW exposure, and conserved PPE supply. Our interventions serve as a model for leveraging the collaborative relationship between pharmacists and antimicrobial stewardship programs during the COVID-19 pandemic. With some modifications to accommodate other institutions' work flows, this initiative can be adapted by other antimicrobial stewardship programs and pharmacy departments. During these challenging times, it is imperative to engage in multidisciplinary collaboration to not only keep the patient safe but our own colleagues as well. We hope our project inspires other creative ways for antimicrobial stewardship programs to contribute to efforts to prevent HCW exposure to SARS-CoV-2.
Acknowledgments.

Financial support. No financial support was provided relevant to this article.

Conflicts of interest. All authors report no conflicts of interest relevant to this article.

\section{References}

1. Stevens MP, Patel PK, Nori P. Involving antimicrobial stewardship programs in COVID-19 response efforts: all hands on deck. Infect Cont Hosp Epidemiol 2020;41:744-745.

2. Stevens RW, Estes L, Rivera C. Practical implementation of COVID-19 patient flags into an antimicrobial stewardship program's prospective review. Infect Cont Hosp Epidemiol 2020;15:1-2.

\title{
Examining the need for eye protection for coronavirus disease 2019 (COVID-19) prevention in the community
}

\author{
Alexandre R. Marra MD, MS ${ }^{1,2,3}$ (1), Michael B. Edmond MD, MPH, MPA², Saskia V. Popescu PhD, MPH, MA, CIC ${ }^{4}$ and \\ Eli N. Perencevich MD, MS ${ }^{1,2}$ \\ ${ }^{1}$ Center for Access \& Delivery Research and Evaluation (CADRE), lowa City VA Health Care System, lowa City, lowa, United States, ${ }^{2}$ Department of Internal \\ Medicine, University of lowa Hospital \& Clinics, lowa City, lowa, United States, ${ }^{3}$ Albert Einstein Jewish Institute for Education and Research, Hospital Israelita \\ Albert Einstein, São Paulo, Brazil and ${ }^{4}$ George Mason University, Fairfax, Virginia, United States
}

To the Editor-As the world reopens after extreme social distancing designed to flatten the curve and protect hospitals, it appears that even countries that had controlled coronavirus disease 2019 (COVID-19) with widespread testing and contact tracing, such as South Korea and Singapore, are seeing increased case counts. One proposed method for reducing transmission as society reopens is requiring the public to wear face coverings, including cotton face masks or face shields. ${ }^{1}$ An important factor that distinguishes face shields from masks is eye protection. Yet the importance of eye protection in the prevention of COVID-19 and other coronaviruses is underappreciated, which has led to public health authorities recommending cotton face masks over potentially more protective alternatives, such as face shields.

The mucous membranes of healthcare workers (HCWs), including the conjunctiva, may be exposed to respiratory droplets from the patient. ${ }^{2}$ The importance of eye protection during care of patients with novel coronaviruses was recognized in 2003 during the severe acute respiratory syndrome coronavirus (SARS-CoV-1) outbreaks and subsequent Middle East respiratory syndrome coronavirus (MERS-CoV) outbreaks. ${ }^{3}$ For example, during SARS, the lack of eye protection when transferring a patient may have been the primary risk factor for one of the first doctors infected. $^{4}$

It has been increasingly recognized that severe acute respiratory coronavirus virus 2 (SARS-CoV-2) can be transmitted from

Author for correspondence: Eli N. Perencevich, E-mail: eli-perencevich@uiowa.edu Cite this article: Marra AR, et al. (2021). Examining the need for eye protection for coronavirus disease 2019 (COVID-19) prevention in the community. Infection Control \& Hospital Epidemiology, 42: 646-647, https://doi.org/10.1017/ice.2020.314 infected individuals when they are asymptomatic or presymptomatic. ${ }^{3,5}$ Thus, to prevent transmission in the community, personal protective equipment (PPE) must be worn at all times in addition to other containment measures such as $2 \mathrm{~m}$ (6 feet) distancing and avoiding large gatherings. Both droplet and contact transmission routes have been implicated in the spread of SARS-CoV-2. ${ }^{1,3}$ PPE has 2 potential benefits when worn in the community: (1) PPE can provide source control by containing the respiratory droplets generated through coughs, sneezes or during speech and (2) PPE can act as a barrier preventing respiratory droplets from landing on facial mucosal membranes or other parts of the face. Additionally, PPE can prevent contact transmission by preventing contaminated hands from reaching the mucosal membranes of the mouth, nose and eyes.

Eye protection might provide additional benefits. A detailed investigation of risk factors for HCW acquisition of SARS, including multivariate generalized estimating equation logistic regression models, identified unprotected eye contact with body fluids as an independent risk factor for infection (odds ratio [OR], 7.34; $P=.001) .{ }^{6}$ However, in a survey of 8 of the 9 US healthcare facilities in which SARS-CoV-1-infected patients were evaluated, $70 \%$ of HCWs reported some exposure to patients without wearing some level of eye protection and none acquired infection. ${ }^{7}$

Although conjunctivitis has been described in a few patients with COVID-19 and other coronavirus syndromes, ${ }^{5}$ emerging evidence supports that coronavirus can enter the host via the conjunctival route. ${ }^{8}$ Conjunctiva may be a potential portal for infection ${ }^{9}$ because it is directly exposed to extraocular pathogens, and the mucosa of the ocular surface and upper respiratory tract are connected by the nasolacrimal duct and have been shown to

(C) 2020 by The Society for Healthcare Epidemiology of America. All rights reserved. This is an Open Access article, distributed under the terms of the Creative Commons Attribution licence (http://creativecommons.org/licenses/by/4.0/), which permits unrestricted re-use, distribution, and reproduction in any medium, provided the original work is properly cited. 
share the same entry receptors for some respiratory viruses, ${ }^{5}$ including angiotensin-converting enzyme 2 (ACE2) for SARS-CoV-1 and SARS-CoV-2. ${ }^{2,5}$ In addition, SARS-CoV-2 was detectable in several nasolacrimal system-associated tissues, including the conjunctiva, lacrimal gland, nasal cavity, and throat, thus validating the anatomical bridge between ocular mucosa and the respiratory tract. ${ }^{8}$ Finally, macaques were susceptable to SARS$\mathrm{CoV}-2$ infection via the conjunctival route and progressed to lung infections suggesting the biological importance of eye infection. ${ }^{10}$

Given that SARS-CoV-2 can be transmitted by fomites and droplets that contact the mucous membranes of the mouth and nose, as well as the eyes, it appears that until proven otherwise, HCWs and at-risk citizens in the community should use barriers to protect their entire face including their eyes. Current public health guidance recommends cotton face masks, but given the potential role of the conjunctival route, face shields that provide barrier protection for the entire face might be the superior option. Further research in this area is critically needed.

\section{Acknowledgments.}

Financial support. All authors report no source of funding or support.

Conflicts of interest. All authors report no conflicts of interest relevant to this article.

\section{References}

1. Perencevich EN, Diekema DJ, Edmond MB. Moving personal protective equipment into the community: face shields and containment of COVID-19. JAMA 2020;323:2252-2253.
2. Khunti $\mathrm{K}$, Greenhalgh $\mathrm{T}$, Chan $\mathrm{XH}$, et al. What is the efficacy of eye protection equipment compared to no eye protection equipment in preventing transmission of COVID-19-type respiratory illness in primary and community care? The Centre for Evidence-Based Medicine website. https://www.cebm.net/covid-19/what-is-the-efficacy-of-eye-protectionequipment-compared-to-no-eye-protection-equipment-in-preventingtransmission-of-covid-19-type-respiratory-illnesses-in-primary-andcommunity-care/. Accessed May 28, 2020.

3. Chou R, Dana T, Buckley DI, Selph S, Fu R, Totten AM. Epidemiology of and risk factors for coronavirus infection in healthcare workers. Ann Intern Med 2020 May 5 [Epub ahead of print]. doi: 10.7326/M201632.

4. Fisher DA, Chew MN, Lim YT, Tambyah PA. Preventing local transmission of SARS: lessons from Singapore. Med J Aust 2003;178:555-558.

5. Sun CB, Wang YY, Liu GH, Liu Z. Role of the eye in transmitting human coronavirus: what we know and what we do not know. Front Public Health 2020;8:155.

6. Raboud J, Shigayeva A, McGeer A, et al. Risk factors for SARS transmission from patients requiring intubation: a multicentre investigation in Toronto, Canada. PLoS One 2010;5(5):e10717.

7. Park BJ, Peck AJ, Kuehnert MJ, et al. Lack of SARS transmission among healthcare workers, United States. Emerg Infect Dis 2004;10:244-248.

8. Siedlecki J, Brantl V, Schworm B, et al. COVID-19: ophthalmological aspects of the SARS-CoV 2 global pandemic. Klin Monbl Augenheilkd 2020;237:675-680.

9. Chen L, Liu M, Zhang Z, et al. Ocular manifestations of a hospitalised patient with confirmed 2019 novel coronavirus disease. Br J Ophthalmol 2020 Apr 7 [Epub ahead of print]. doi: 10.1136/bjophthalmol2020-316304.

10. Deng W, Bao L, Gao H, et al. Ocular conjunctival inoculation of SARS-CoV-2 can cause mild COVID-19 in Rhesus macaques. bioRxiv; 2020. doi: 10.1101/ 2020.03.13.990036.

\title{
Limited impact of selective susceptibility reporting of Escherichia coli and Klebsiella isolates from concurrent blood and urine cultures
}

\author{
Brenton C. Hall PharmD ${ }^{1, *}$, Julie S. Alexander DO ${ }^{2, \star}$, Shelby S. Anderson PharmD ${ }^{1}$, Jessica K. Ortwine PharmD, BCIDP ${ }^{1,2}$, \\ Norman S. Mang PharmD, BCIDP ${ }^{1,2}$, Wenjing Wei PharmD, BCPS ${ }^{1,2}$, Linda S. Hynan PhD ${ }^{3}$ and Bonnie C. Prokesch MD² (1) \\ ${ }^{1}$ Department of Pharmacy, Parkland Health \& Hospital System, Dallas, Texas, ${ }^{2}$ Department of Medicine, University of Texas Southwestern Medical Center, Dallas, \\ Texas and ${ }^{3}$ Department of Clinical Sciences, University of Texas Southwestern Medical Center, Dallas, Texas
}

To the Editor-In 2019, the Centers for Disease Control and Prevention reported that $>2.8$ million antibiotic-resistant infections occur in the United States annually, with nearly 35,000 deaths as a result. ${ }^{1}$ One method of minimizing the emergence of antimicrobial resistance is through antimicrobial stewardship. The Infectious Diseases Society of America has published guidelines on antimicrobial stewardship identifying potential interventions to guide appropriate antimicrobial prescribing, which includes selective or cascade reporting of antibiotic susceptibility data. ${ }^{2}$ In cascade reporting, specific antibiotics in the susceptibility report

Author for correspondence: Bonnie C. Prokesch, E-mail: bonnie.prokesch@ utsouthwestern.edu

${ }^{\star}$ Authors of equal contribution.

Cite this article: Hall BC, et al. (2021). Limited impact of selective susceptibility reporting of Escherichia coli and Klebsiella isolates from concurrent blood and urine cultures. Infection Control \& Hospital Epidemiology, 42: 647-648, https://oi.org/ $10.1017 /$ ice. 2020.308 are deliberately withheld from the view of clinicians when the organism is susceptible to more narrow-spectrum agents. ${ }^{3}$ Because of the limited data on clinical outcomes, cascade reporting is classified as a weak recommendation. ${ }^{2}$

In 2016, antimicrobial data at our 862-bed county hospital in Dallas, Texas, revealed piperacillin-tazobactam (PT) as the most utilized broad-spectrum gram-negative antimicrobial, with an average of 103 days of therapy per 1,000 patient days. During the same time, 11,306 isolates from the Enterobacterales family were identified from various sources, with $85 \%$ being Escherichia coli or Klebsiella spp. The objective of this retrospective study was to determine whether a cascade reporting system influenced the de-escalation of empiric PT in patients with E. coli and Klebsiella bacteremia due to a urinary source and subsequent effects on patient outcomes.

On September 7, 2017, the clinical microbiology laboratory implemented a cascading antibiotic algorithm for non-extended- 